\title{
AN IMPROVED MENSHOV-RADEMACHER THEOREM
}

\author{
FERENC MÓRICZ AND KÁROLY TANDORI
}

(Communicated by J. Marshall Ash)

\begin{abstract}
We study the a.e. convergence of orthogonal series defined over a general measure space. We give sufficient conditions which contain the Menshov-Rademacher theorem as an endpoint case. These conditions turn out to be necessary in the particular case where the measure space is the unit interval $[0,1]$ and the moduli of the coefficients form a nonincreasing sequence. We also prove a new version of the Menshov-Rademacher inequality.
\end{abstract}

\section{INTRODUCTION}

In this note we consider an arbitrary measure space $X$ with measure $\mu$. We denote by $\Omega$ the class of orthonormal systems $\varphi:=\left\{\varphi_{k}(x): k \geq 1, x \in X\right\}$ of functions. That is, we always assume that

$$
\int \varphi_{k}(x) \varphi_{l}(x) d \mu(x)=0 \quad(k \neq l), \quad \int \varphi_{k}^{2}(x) d \mu(x)=1 \quad(k, l=1,2, \ldots) .
$$

Here and in the sequel, the integrals are extended over the whole space $X$.

Given a sequence $a=\left\{a_{k}: k \geq 1\right\}$ of real numbers we introduce the quantity

$$
\|a(m, n)\|:=\sup \left\{\int\left(\max _{m \leq p \leq n}\left|\sum_{k=m}^{p} a_{k} \varphi_{k}(x)\right|\right)^{2} d \mu(x)\right\}^{1 / 2},
$$

where $m, n$ are integers, $1 \leq m \leq n$, and the supremum is taken over all systems $\varphi \in \Omega$. Furthermore, set

$$
\|a\|:=\lim _{n \rightarrow \infty}\|a(1, n)\|
$$

which may be infinite.

The following theorem proved by the second named author characterizes those sequences $a$ for which the orthogonal series

$$
\sum_{k=1}^{\infty} a_{k} \varphi_{k}(x)
$$

converges a.e. for all $\varphi \in \Omega$.

Received by the editors November 1, 1993 and, in revised form, September 26, 1994

1991 Mathematics Subject Classification. Primary 42C05.

Key words and phrases. Orthonormal system, a.e. convergence, Menshov-Rademacher inequality and theorem.

This research was partially supported by the Hungarian National Foundation for Scientific Research under Grant \#234. 
Theorem A ([8, Theorem 1]). The orthogonal series (1.1) converges a.e. for all $\varphi \in \Omega$ if and only if $\|a\|<\infty$.

In general, it is a difficult task to check whether the norm $\|a\|$ is finite or not. Therefore it makes sense to look for necessary or sufficient conditions, which are easier to apply in concrete situations.

\section{Sufficient CONDitions}

The well-known Menshov-Rademacher theorem (see [2], [5], and also [1, p. 80]) states that if

$$
\sum_{k=1}^{\infty} a_{k}^{2}(\log k)^{2}<\infty
$$

then the orthogonal series (1.1) converges a.e. for all $\varphi \in \Omega$. Here and in the sequel, the logarithms are to the base 2 .

We note that condition (2.1) is the best possible in the sense that for any nondecreasing sequence $\{w(k)\}$ of positive numbers such that

$$
w(k)=o(\log k) \quad(k \rightarrow \infty)
$$

there exist a sequence $\left\{a_{k}\right\}$ of real numbers and a particular system $\varphi \in \Omega$ such that

$$
\sum_{k=1}^{\infty} a_{k}^{2} w^{2}(k)<\infty
$$

but the orthogonal series (1.1) diverges a.e. (see, e.g., [1, p. 88]).

The second named author improved the above result by showing (see [9]) that if

$$
\sum_{k=1}^{\infty} a_{k}^{2}(\log k) \log _{+} \frac{1}{a_{k}^{2}}<\infty
$$

then the orthogonal series (1.1) converges a.e. for all $\varphi \in \Omega$. Here we agree to put

$$
\log _{+}\left(1 / a_{k}^{2}\right)= \begin{cases}\log \left(1 / a_{k}^{2}\right) & \text { if } 0<a_{k}^{2} \leq 1 / 2 \\ 1 & \text { if } a_{k}^{2}>1 / 2 \text { or } a_{k}=0 .\end{cases}
$$

In [4] we proved that condition (2.2) can be replaced by the somewhat better condition

$$
\sum_{r=0}^{\infty} \sum_{k \in J_{r}} a_{k}^{2}(\log k) \log \left(\frac{2}{a_{k}^{2}} \sum_{l \in J_{r}} a_{l}^{2}\right)<\infty,
$$

where

$$
J_{r}:=\left\{n_{r}+1, n_{r}+2, \ldots, n_{r+1}\right\} \quad \text { and } \quad n_{r}:=2^{2^{r}} \quad(r \geq 0) .
$$

It is easy to see that condition (2.3) follows from (2.2), and condition (2.2) follows from (2.1), but not conversely.

Before stating the main result of this paper, we introduce the following notations:

$$
I_{\nu}:=\left\{2^{\nu}+1,2^{\nu}+2, \ldots, 2^{\nu+1}\right\}, \quad A_{\nu}^{2}:=\sum_{k \in I_{\nu}} a_{k}^{2} \quad(\nu \geq 0),
$$

and we agree to put $a_{k}^{2} \log \left(2 A_{\nu}^{2} / a_{k}^{2}\right)=0$ in case $a_{k}=0$. 
Theorem 1. If for some $0<\varepsilon \leq 2$ we have

$$
\sum_{\nu=0}^{\infty} \sum_{k \in I_{\nu}} a_{k}^{2}(\log k)^{\varepsilon}\left(\log \frac{2 A_{\nu}^{2}}{a_{k}^{2}}\right)^{2-\varepsilon}<\infty
$$

then the orthogonal series (1.1) converges a.e. for all $\varphi \in \Omega$.

It turns out that even more is true. Namely, under (2.6), the rearranged series

$$
\sum_{j=1}^{\infty} a_{k_{j}} \varphi_{k_{j}}(x)
$$

converges a.e. for all $\varphi \in \Omega$ and for all (so-called weak) permutations $\left\{k_{1}, k_{2}, \ldots\right\}$ of the positive integers $\{1,2, \ldots\}$ such that $\left\{k_{j}: j \in I_{\nu}\right\}$ is a permutation of $I_{\nu}$ in the case of $\nu$ large enough.

It is instructive to consider the special case of (2.6) corresponding to $\varepsilon=1$ :

$$
\sum_{\nu=0}^{\infty} \sum_{k \in I_{\nu}} a_{k}^{2}(\log k) \log \frac{2 A_{\nu}^{2}}{a_{k}^{2}}<\infty .
$$

We claim that even this particular condition is better than (2.3). Indeed, a simple estimation gives

$$
\begin{aligned}
& \sum_{\nu=1}^{\infty} \sum_{k \in I_{\nu}} a_{k}^{2}(\log k) \log \frac{2 A_{\nu}^{2}}{a_{k}^{2}} \\
& \quad=\sum_{r=0}^{\infty} \sum_{\nu=2^{r}}^{2^{r+1}-1} \sum_{k \in I_{\nu}} a_{k}^{2}(\log k) \log \frac{2 A_{\nu}^{2}}{a_{k}^{2}} \\
& \leq \sum_{r=0}^{\infty} \sum_{k \in J_{r}} a_{k}^{2}(\log k) \log \left(\frac{2}{a_{k}^{2}} \sum_{l \in J_{r}} a_{l}^{2}\right) \\
& =\sum_{r=0}^{\infty} \sum_{k \in J_{r}} a_{k}^{2}(\log k) \log \left(\frac{2}{a_{k}^{2}} \sum_{l \in J_{r}} a_{l}^{2}\right)
\end{aligned}
$$

which justifies our claim.

In order to prove Theorem 1, first we prove a new version of the famous MenshovRademacher inequality (see, e.g., [1, p. 79] and [3, Theorem 3]), which says

$$
\int\left(\max _{1 \leq n \leq N}\left|\sum_{k=1}^{n} a_{k} \varphi_{k}(x)\right|\right)^{2} d \mu(x) \leq(\log 2 N)^{2} \sum_{k=1}^{N} a_{k}^{2} \quad(N \geq 1) .
$$

Lemma 1. For every $\varepsilon, 0<\varepsilon \leq 2$, there exists a constant $C$ depending only on $\varepsilon$ such that for all sequences $a$, all $\varphi \in \Omega$, and all integers $N \geq 1$, we have

$$
\begin{aligned}
& \int\left(\max _{1 \leq n \leq N}\left|\sum_{k=1}^{n} a_{k} \varphi_{k}(x)\right|\right)^{2} d \mu(x) \\
& \quad \leq C(\log 2 N)^{\varepsilon} \sum_{k=1}^{N} a_{k}^{2}\left(\log \frac{2 A^{2}}{a_{k}^{2}}\right)^{2-\varepsilon}, \quad A^{2}:=\sum_{k=1}^{N} a_{k}^{2} .
\end{aligned}
$$


Proof of Lemma 1. Inequality (2.10) is obvious in case $N=1$ and 2, provided $C \geq 2$. In case $N \geq 3$, we may assume that

$$
N=n_{r}:=2^{2^{r}} \quad \text { for some } r \geq 1 .
$$

Otherwise, we may supplement the given coefficients $a_{1}, a_{2}, \ldots, a_{N}$ by an appropriate number of zeros, which does not affect the left-hand side in (2.10), while the right-hand side increases at most by $2^{\varepsilon}$.

We rearrange the $\left|a_{k}\right|$ in a descending order of magnitude:

$$
\left|a_{d(1)}\right| \geq\left|a_{d(2)}\right| \geq \cdots \geq\left|a_{d\left(n_{r}\right)}\right| .
$$

For each $p=0,1, \ldots, r-1$, we consider the integers of the block $\left\{d(k): k \in J_{p}\right\}$ in their original (increasing) order, where $J_{p}$ is defined in (2.4). More precisely, let $\left\{e(k): k \in J_{p}\right\}$ be the permutation of $J_{p}$ such that

$$
d\left(e\left(n_{p}+1\right)\right)<d\left(e\left(n_{p}+2\right)\right)<\cdots<d\left(e\left(n_{p+1}\right)\right) .
$$

Our key estimate is the following:

$$
\begin{aligned}
M(x) & :=\max _{1 \leq n \leq N}\left|\sum_{k=1}^{n} a_{k} \varphi_{k}(x)\right| \\
& \leq\left|a_{d(1)} \varphi_{d(1)}(x)\right|+\left|a_{d(2)} \varphi_{d(2)}(x)\right|+\sum_{p=0}^{r-1} \max _{n \in J_{p}}\left|\sum_{k=n_{p}+1}^{n} a_{d(e(k))} \varphi_{d(e(k))}(x)\right|
\end{aligned}
$$

Applying Minkowski's inequality and (2.9) gives

$$
\left\{\int M^{2}(x) d \mu(x)\right\}^{1 / 2} \leq\left|a_{d(1)}\right|+\left|a_{d(2)}\right|+\sum_{p=0}^{r-1}\left(\log 2 n_{p+1}\right)\left\{\sum_{k \in J_{p}} a_{d(k)}^{2}\right\}^{1 / 2}
$$

By the Cauchy-Schwarz inequality, we obtain

$$
\begin{aligned}
& \sum_{p=0}^{r-1}\left\{\sum_{k \in J_{p}} a_{d(k)}^{2}(\log 2 k)^{2}\right\}^{1 / 2} \\
& \leq 4^{\varepsilon / 2} \sum_{p=0}^{r-1}\left\{2^{p \varepsilon} \sum_{k \in J_{p}} a_{d(k)}^{2}(\log 2 k)^{2-\varepsilon}\right\}^{1 / 2} \\
& \leq 4^{\varepsilon / 2}\left\{\sum_{p=0}^{r-1} 2^{p \varepsilon}\right\}^{1 / 2}\left\{\sum_{p=0}^{r-1} \sum_{k \in J_{p}} a_{d(k)}^{2}(\log 2 k)^{2-\varepsilon}\right\}^{1 / 2} \\
& \quad \leq \frac{4^{\varepsilon / 2}}{\left(2^{\varepsilon}-1\right)^{1 / 2}}\left\{2^{r \varepsilon} \sum_{k=3}^{n_{r}} a_{d(k)}^{2}(\log 2 k)^{2-\varepsilon}\right\}^{1 / 2} .
\end{aligned}
$$

By (2.12), we have $k a_{d(k)}^{2} \leq A^{2}$ for all $k$. Thus,

$$
\sum_{k=3}^{n_{r}} a_{d(k)}^{2}(\log 2 k)^{2-\varepsilon} \leq \sum_{k=3}^{n_{r}} a_{d(k)}^{2}\left(\log \frac{2 A^{2}}{a_{d(k)}^{2}}\right)^{2-\varepsilon}
$$


Taking into account (2.11), (2.13)-(2.15), and the fact that $\log \left(2 A^{2} / a_{d(k)}^{2}\right) \geq 1$, we conclude

$$
\begin{aligned}
& \left\{\int M^{2}(x) d \mu(x)\right\}^{1 / 2} \\
& \quad \leq\left(2 \frac{4^{\varepsilon / 2}}{\left(2^{\varepsilon}-1\right)^{1 / 2}}+2\right)\left\{(\log N)^{\varepsilon} \sum_{k=1}^{n_{r}} a_{d(k)}^{2}\left(\log \frac{2 A^{2}}{a_{d(k)}^{2}}\right)^{2-\varepsilon}\right\}^{1 / 2} .
\end{aligned}
$$

This proves (2.10), since the right-hand side is symmetric in the terms $a_{1}, a_{2}, \ldots$, $a_{n_{r}}$.

Denote by

$$
s_{n}(x):=\sum_{k=1}^{n} a_{k} \varphi_{k}(x)
$$

the $n$th partial sum of series (1.1).

Lemma 2 (see, e.g., [1, p. 83]). If

$$
\sum_{k=2}^{\infty} a_{k}^{2}(\log \log k)^{2}<\infty
$$

then the subsequence $\left\{s_{2^{\nu}}(x): \quad \nu \geq 0\right\}$ of the partial sums converges a.e. for all $\varphi \in \Omega$.

Proof of Theorem 1. From (2.6) it follows that

$$
\sum_{k=2}^{\infty} a_{k}^{2}(\log k)^{\varepsilon}<\infty
$$

which in turn implies (2.16). By Lemma $2, s_{2^{\nu}}(x)$ converges a.e. as $m \rightarrow \infty$.

It remains to estimate the maximal fluctuation

$$
M_{\nu}(x):=\max _{n \in I_{\nu}}\left|\sum_{k=2^{\nu}+1}^{n} a_{k} \varphi_{k}(x)\right| \quad(\nu \geq 1) .
$$

By Lemma 1,

$$
\int M_{\nu}^{2}(x) d \mu(x) \leq C(\nu+1)^{\varepsilon} \sum_{k \in I_{\nu}} a_{k}^{2}\left(\log \frac{2 A_{\nu}^{2}}{a_{k}^{2}}\right)^{2-\varepsilon}
$$

By (2.6),

$$
\sum_{\nu=1}^{\infty} \int M_{\nu}^{2}(x) d \mu(x)<\infty
$$

whence, by the dominated convergence theorem,

$$
\lim _{\nu \rightarrow \infty} \max _{n \in I_{\nu}}\left|\sum_{k=2^{\nu}+1}^{n} a_{k} \varphi_{k}(x)\right|=0 \quad \text { a.e. }
$$

This completes the proof of Theorem 1 . 


\section{NECESSARY CONDITIONS}

From now on, let the measure space $X$ be the unit interval $[0,1]$ with Borel measurable subsets and Lebesgue measure $\mu$. Clearly, the condition

$$
\sum_{k=1}^{\infty} a_{k}^{2}<\infty
$$

is necessary for the orthogonal series (1.1) to converge a.e. for all $\varphi \in \Omega$. To see this, it is enough to take the Rademacher system in the capacity of $\varphi$ (see, e.g., [1, p. 54]).

The second named author gave the following nontrivial necessary condition.

Theorem B ([6, Theorem 1]). If the orthogonal series (1.1) converges a.e. for all $\varphi \in \Omega$, then

$$
\sum_{k=1}^{\infty} a_{k}^{2}\left(\log _{+} \frac{1}{a_{k}^{2}}\right)^{2}<\infty .
$$

It is plain that (3.1) implies

$$
\sum_{\nu=0}^{\infty} \sum_{k \in I_{\nu}} a_{k}^{2}\left(\log \frac{2 A_{\nu}^{2}}{a_{k}^{2}}\right)^{2}<\infty
$$

This latter condition coincides with (2.6) for $\varepsilon=0$.

We note that condition (3.1) is not sufficient in general for the orthogonal series (1.1) to converge a.e. for all $\varphi \in \Omega$. This is shown by the next

Example 1. The second named author proved in [7] that if $\left|a_{1}\right| \geq\left|a_{2}\right| \geq \cdots$, then all rearrangements (2.7) of the orthogonal series (1.1) converge a.e. for all $\varphi \in \Omega$ if and only if

$$
\sum_{r=0}^{\infty}\left\{\sum_{k \in J_{r}} a_{k}^{2}(\log k)^{2}\right\}^{1 / 2}<\infty
$$

where the blocks $J_{r}$ are defined by (2.4).

Now, for the sequence

$$
a_{k}:=r^{-1} 2^{-r}\left(n_{r+1}-n_{r}\right)^{-1 / 2} \quad \text { if } k \in J_{r}(r \geq 0)
$$

condition (3.1) is satisfied, but (3.3) is not. Consequently, there exist a system $\left\{\varphi_{k}\right\} \in \Omega$ on the unit interval $[0,1]$ and a permutation $\left\{k_{j}\right\}$ of the positive integers such that the rearranged series (2.7) diverges a.e. (see [7]). It remains to observe that condition (3.1) is invariant under a permutation of its terms.

On the other hand, condition (2.6) for some $\varepsilon>0$ is not necessary for the orthogonal series (1.1) to converge a.e. for all $\varphi \in \Omega$. To see this, we present the following

Example 2. Let

$$
a_{k}:= \begin{cases}r^{-2} & \text { if } k=n_{r}:=2^{2^{r}} \quad(r \geq 1) \\ 0 & \text { otherwise }\end{cases}
$$


Then condition (2.6) is not satisfied for any $\varepsilon>0$. Nevertheless, series (1.1) converges a.e. for all $\varphi \in \Omega$, due to

$$
\sum_{k=1}^{\infty}\left|a_{k}\right|=\sum_{r=1}^{\infty} r^{-2}<\infty
$$

\section{Analysis of COndition (2.6)}

Given a sequence $a:=\left\{a_{k}: k \geq 1\right\}$ of real numbers, consider the series occurring in condition (2.6) and denote its sum by $\sum_{\varepsilon}$ for some $\varepsilon \geq 0$. That is, let

$$
\sum_{\varepsilon}:=\sum_{\nu=0}^{\infty} \sum_{k \in I_{\nu}} a_{k}^{2}(\log k)^{\varepsilon}\left(\log \frac{2 A_{\nu}^{2}}{a_{k}^{2}}\right)^{2-\varepsilon},
$$

which may be infinite. We note that condition (2.6) reduces to (2.1) if $\varepsilon=2$, to (2.8) if $\varepsilon=1$, and to (3.2) if $\varepsilon=0$.

We shall prove the following

Theorem 2. Let $0 \leq \delta<\varepsilon \leq 2$. Then there exists an absolute constant $C_{1}$ such that for every sequence $a=\left\{a_{k}\right\}$

$$
\sum_{\delta} \leq C_{1} \sum_{\varepsilon}
$$

If the sequence $\left\{\left|a_{k}\right|\right\}$ is nonincreasing, then the converse inequality

$$
\sum_{\varepsilon} \leq C_{2} \sum_{\delta}
$$

also holds, with another absolute constant $C_{2}$.

Combining Theorems 1 and 2 with Theorem B provides the following known characterization (see, e.g., [8] and [9]).

Corollary. If a sequence $\left\{\left|a_{k}\right|\right\}$ is nonincreasing, then conditions (2.1), (2.8), and (3.2) are pairwise equivalent, and each of them is necessary and sufficient for the orthogonal series (1.1) to converge a.e. for all $\varphi \in \Omega$.

Before proving Theorem 2, we prove a simplified version for finite sequences.

Lemma 3. There exists an absolute constant $C_{3}$ such that for all sequences $a=$ $\left\{a_{k}\right\}$ and all $N \geq 1$ we have

$$
\sum_{k=1}^{N} a_{k}^{2}\left(\log \frac{2 A^{2}}{a_{k}^{2}}\right)^{2} \leq C_{3} \sum_{k=1}^{N} a_{k}^{2}(\log 2 k)^{2}, \quad A^{2}:=\sum_{k=1}^{N} a_{k}^{2} .
$$

If the sequence $\left\{\left|a_{k}\right|\right\}$ is nonincreasing, then

$$
\sum_{k=1}^{N} a_{k}^{2}(\log 2 k)^{2} \leq \sum_{k=1}^{N} a_{k}^{2}\left(\log \frac{2 A^{2}}{a_{k}^{2}}\right)^{2} .
$$

Proof of Lemma 3. First, we assume that the sequence $\left\{\left|a_{k}\right|\right\}$ is nonincreasing. Without loss of generality, we may assume that $A=1$. By monotonicity,

$$
k a_{k}^{2} \leq 1, \quad \text { whence } 2 k \leq 2 / a_{k}^{2}(1 \leq k \leq N) .
$$

This proves (4.4). 
From now on, we do not use monotonicity of $\left\{\left|a_{k}\right|\right\}$. We shall distinguish between two cases accordingly as

(i) $k^{4} a_{k}^{2} \geq 1$, whence $\log \frac{2}{a_{k}^{2}} \leq 4 \log 2 k$;

(ii) $k^{4} a_{k}^{2}<1$, whence $a_{k}^{2}\left(\log \frac{2}{a_{k}^{2}}\right) \leq \frac{C_{4}}{k^{2}}$,

where

$$
C_{4}:=\max _{0<t \leq 1} t\left(\log \frac{2}{t^{2}}\right)^{2}
$$

To sum up,

$$
\sum_{k=1}^{N} a_{k}^{2}\left(\log \frac{2}{a_{k}^{2}}\right)^{2} \leq 16 \sum_{k=1}^{N} a_{k}^{2}(\log 2 k)^{2}+C_{4} \sum_{k=1}^{N} \frac{1}{k^{2}} .
$$

Hence (4.3) follows with $C_{3}:=16+C_{4} \frac{\pi^{2}}{6}$.

Proof of Theorem 2. It is plain that if $\sum_{\varepsilon}<\infty$ for some $\varepsilon>0$, then

$$
\mathcal{A}:=\sum_{k=1}^{\infty} a_{k}^{2}<\infty
$$

Without loss of generality, we may assume that $\mathcal{A}=1$.

Again, we distinguish between two cases accordingly as

(i) $k^{4} a_{k}^{2} \geq A_{\nu}^{2}$, whence $\log \frac{2 A_{\nu}^{2}}{a_{k}^{2}} \leq 4 \log 2 k$;

(ii) $k^{4} a_{k}^{2}>A_{\nu}^{2}$, whence $a_{k}^{2}\left(\log \frac{2 A_{\nu}^{2}}{a_{k}^{2}}\right)^{\varepsilon-\delta} \leq C_{4} \frac{A_{\nu}}{k^{2}}$,

where $C_{4}$ is defined in (4.5). Hence

$$
\begin{aligned}
\sum_{\delta} \leq & 4^{\varepsilon-\delta} \sum_{\nu=0}^{\infty} \sum_{k \in I_{\nu}} a_{k}^{2}(\log 2 k)^{\varepsilon}\left(\log \frac{2 A_{\nu}^{2}}{a_{k}^{2}}\right)^{2-\varepsilon} \\
& +C_{4} \sum_{\nu=0}^{\infty} \sum_{k \in I_{\nu}} \frac{(\log 2 k)^{\delta}}{k^{2}} A_{\nu} .
\end{aligned}
$$

Since $A_{\nu} \leq \mathcal{A}=1$, we get

$$
\sum_{\delta} \leq 16 \sum_{\varepsilon}+C_{4} \sum_{k=2}^{\infty} \frac{(\log 2 k)^{2}}{k^{2}} .
$$

Due to the fact that $\sum_{\varepsilon} \geq \mathcal{A}^{2}=1$, this gives (4.1) with

$$
C_{1}:=16+C_{4} \sum_{k=2}^{\infty} \frac{(\log 2 k)^{2}}{k^{2}} .
$$

Relying on (4.1) just proved, it is enough to check (4.2) in the special case when $\delta=0$ and $\varepsilon=2$. By Lemma 3 , for $\nu \geq 6$ we have

$$
\begin{aligned}
\frac{1}{2} \sum_{k=2^{\nu}+2^{\nu-1}}^{2^{\nu+1}} a_{k}^{2}(\log 2 k)^{2} & \leq \sum_{k=2^{\nu}+2^{\nu-1}}^{2^{\nu+1}} a_{k}^{2}\left(\log 2\left(k-2^{\nu}\right)\right)^{2} \\
& \leq \sum_{k \in I_{\nu}} a_{k}^{2}\left(\log \frac{2 A_{\nu}^{2}}{a_{k}^{2}}\right)^{2} .
\end{aligned}
$$


By monotonicity, for $\nu \geq 6$ we have

$$
\frac{1}{8} \sum_{k \in I_{\nu+1}} a_{k}^{2}(\log 2 k)^{2} \leq \sum_{k=2^{\nu}+2^{\nu-1}}^{2^{\nu+1}} a_{k}^{2}(\log 2 k)^{2} .
$$

Combining (4.6) and (4.7) yields (4.2).

\section{ACKNOWLEDGMENT}

The authors are grateful to the referee for the valuable suggestions which improved the presentation of this paper.

\section{REFERENCES}

1. G. Alexits, Convergence problems of orthogonal series, Hungarian Acad. Sci., Budapest, 1961. MR 36:1911

2. D. E. Menchoff, Sur les séries des fonctions orthogonales (Première partie), Fund. Math. 4 (1923), 92-105.

3. F. Móricz, Moment inequalities and the strong laws of large numbers, Z. Wahrsch. Verw. Gebiete 35 (1976), 299-314. MR 53:11717

4. F. Móricz and K. Tandori, Almost everywhere convergence of orthogonal series revisited, J. Math. Anal. Appl. 182 (1994), 637-653. MR 95a:42049

5. H. Rademacher, Einige Sätze über Reihen von allgemeinen Orthogonalfunktionen, Math. Ann. 87 (1922), 112-138.

6. K. Tandori, Über die Divergenz der Orthogonalreihen, Publ. Math. Debrecen 8 (1961), 291307. MR 25:2374

7. __ Orthogonalen Funktionen X, Acta Sci. Math. (Szeged) 23 (1962), 185-221. MR 26: 1688

8. — Über die Konvergenz der Orthogonalreihen II, Acta Sci. Math. (Szeged) 25 (1964), 219-232. MR 30:1350

9. ——, Bemerkung zur Konvergenz der Orthogonalreihen, Acta Sci. Math. (Szeged) 26 (1965), 249-251. MR 33:3041

Bolyai Institute, University of Szeged, Aradi Vértanúk Tere 1, 6720 Szeged, Hungary E-mail address: moricz@math.u-szeged.hu 